

\section{Quaestio 6/2006}

Annuario di storia della metafisica Annuaire d'histoire de la métaphysique Jahrbuch für die Geschichte der Metaphysik Yearbook of the History of Metaphysics 
(C) 2006, Brepols Publishers n.v., Turnhout, Belgium

(C) 2006, Pagina soc. coop., Bari, Italy

Questo volume è stampato con un contributo

del Consiglio di Amministrazione

dell'Università degli Studi di Bari

All rights reserved. No part of this

publication may be reproduced, stored in a retrieval system, or transmitted, in any form or by any means, electronic, mechanical, photocopying, recording, or otherwise,

without prior permission of the publisher.

ISBN 978-2-503-51980-7

ISSN 1379-2547

D/2007/0095/128 


\section{Quaestio 6/2006}

Agostino e la tradizione agostiniana

Augustin et la tradition augustinienne

Augustinus und die augustinische Tradition

Augustine and the Augustinian Tradition

a cura di

Costantino Esposito e Pasquale Porro

BREPOLS

pagina 


\section{Direzione}

Costantino Esposito e Pasquale Porro

Università di Bari

\section{Comitato Scientifico / Comité Scientifique /}

Wissenschaftlicher Beirat / Advisory Board

Jean-Robert Armogathe (École Pratique des Hautes Études - Paris) - Werner Beierwaltes (München) • Giulia Belgioioso (Lecce) • Enrico Berti (Padova) • Olivier Boulnois (École Pratique des Hautes Études - Paris) - Mario Caimi (Buenos Aires) • Vincent Carraud (Caen) • Mário Santiago de Carvalho (Coimbra) • Jean-François Courtine (Paris IV - Sorbonne) - Kent Emery, Jr. (Notre Dame) • Jorge Gracia (State University of New York - Buffalo) - Miguel Angel Granada (Barcelona) • Dimitri Gutas (Yale) - Friedrich-Wilhelm von Herrmann (Freiburg i.Br.) • Norbert Hinske (Trier) • Ruedi Imbach (Paris IV - Sorbonne) • Ada Lamacchia (Bari) • Alfonso Maierù (Roma «La Sapienza») • Jean-Luc Marion (Paris IV - Sorbonne) • Jean-Marc Narbonne (Laval)

- Dominik Perler (Humboldt-Universität - Berlin) - Gregorio Piaia (Padova) - Stefano Poggi (Firenze) - Paolo Ponzio (Bari) - Riccardo Pozzo (Verona) • Giovanni Reale (Università Vita-Salute San Raffaele - Milano) - Jacob Schmutz (Paris IV - Sorbonne) - William Shea (Padova) • Andreas Speer (Köln) • Carlos Steel (Leuven) • Loris Sturlese (Lecce) • Franco Volpi (Padova)

\section{Redazione}

Anna Arezzo - Marienza Benedetto • Giovanna D'Aniello • Giambattista Formica • Marialucrezia Leone - Marco Lamanna - Vincenzo Lomuscio • Francesca Lunanova - Francesco Marrone • Lucrezia Iris Martone • Stefania Scardicchio • Michele Trizio

Manoscritti e volumi per recensione vanno inviati alla Direzione di «Quaestio»:

Costantino Esposito • Pasquale Porro

Dipartimento di Scienze Filosofiche - Università di Bari

Palazzo Ateneo - Piazza Umberto I

I-70121 - Bari (Italia)

e-mail: esposito@filosofia.uniba.it

porro@filosofia.uniba.it

Abbonamenti / Abonnements / Subscriptions

Brepols Publishers

Begijnhof 67 - B-2300 Turnhout (Belgium)

tel. +32 14 448020 - fax +32 14 428919

e-mail: info.publishers@brepols.com 


\section{Indice}

\section{Agostino e la tradizione agostiniana}

Costantino Esposito / Pasquale Porro (Bari)

Premessa

Giovanni Catapano (Padova)

Quale scetticismo viene criticato da Agostino nel Contra Academicos?

Luigi Alici (Macerata)

Initium omnis operis verbum. On the Semantics of opus/operari in Augustine

Christian Trottmann (Tours)

La Trinité de la Sagesse: contemplation philosophique et guérison mystique

Maria Bettetini (IULM - Milano)

Agostino e l'estetica: un punto

Norbert Fischer (Eichstätt)

Deum et animam scire cupio. Zum bipolaren Grundzug

von Augustins metaphysischem Fragen

Massimo Borghesi (Perugia)

Il daímon o Cristo? La demonologia antica e la critica di Agostino

al demone "mediatore" tra umano e divino

Michele Trizıo (Köln / Bari)

"Un uomo sapiente ed apostolico". Agostino a Bisanzio:

Gregorio Palamas lettore del De trinitate

John A. Demetracopoulos (Patras / Köln)

The Sitz im Leben of Demetrius Cydones' Translation

of pseudo-Augustine's Soliloquia. Remarks on a Recent Edition

ERIC L. SAAK (IUPUI - Indianapolis)

The Episcopacy of Christ: Augustinus of Ancona, OESA

and Political Augustinianism in the Later Middle Ages 
Lydia Wegener (Köln)

Augustinus-Rezeption in der Reformation. Der Straßburger Münsterprediger

Caspar Hedio als Übersetzer augustinischer Schriften in der ersten Hälfte

des 16. Jahrhunderts

Costantino Esposito (Bari)

Da Descartes ad Agostino

Martin W.F. Stone (Leuven)

The Antiquarian and the Moderniser: Giovanni Lorenzo Berti (1696-1766), Pietro Tamburini (1737-1827), and Contrasting Defenses of the Augustinian

Teaching on Unbaptised Infants in Eighteenth-Century Italy

Andrea Staiti (Milano)

Il luogo della verità. La presenza di Agostino nella fenomenologia di Husserl

Aníbal Fornari (Santa Fe, Ar)

San Agustín y la conciencia de la experiencia cultural latinoamericana, en Alberto Rougès

Giusi Strummiello (Bari)

La confessione come pratica filosofica. María Zambrano lettrice di Agostino

Gareth Matthews (Amherst, Mass)

Augustine and Plantinga on the Problem of Evil

Emmanuel Housset (Caen)

Linvention de la personne par saint Augustin et la métaphysique contemporaine

Andreas Grossmann (Hamburg)

Ewiger Friede? Nachaugustinische Retraktationen

483

\section{Varia. Note Cronache Recensioni}

ILARIA RAMELLI

La nuova traduzione della Summa Pitagorica di Giamblico

499

Roberto Schiavolin

L'Uno neoplatonico e la crisi dell'ontologia classica

IVAN A. LICGIARDI

Sulle radici della teologia negativa

Veneranda Castellano

Tempo, computus e conoscenza di Dio nell'Alto Medioevo

Veneranda Castellano / Anna Arezzo

Anselmo di Canterbury e la dialettica

Adriano Oliva

La questione dell'alia lectura di Tommaso d'Aquino. A proposito dell'edizione delle note marginali del ms. Oxford, Lincoln College Lat. 95 
JACOB SсHмUTZ

La naissance de la métaphysique de l'esprit

Cesare Alberto Musatti

Il De caelo di Aristotele e alcuni suoi commentatori:

Simplicio, Averroè e Pietro d'Alvernia

Anna Arezzo

I Quodlibeta teologici del XIII secolo: un contributo alla conoscenza del pensiero medievale

Marco Lamanna

Sulla prima occorrenza del termine «Ontologia». Una nota bibliografica

Guido Cusinato

Spinoza e la mente affettiva

Giovanna D'Aniello

Teologia, darwinismo, nichilismo: la via nietzscheana

Stefania Scardicchio

Il problema dell'esperienza percettiva. Nuove tendenze interpretative

582

Marco SGarbi

Theories of Judgment. Historical and Theoretical Perspectives

Indice dei nomi 


\section{Emmanuel Housset}

\section{L'invention de la personne par saint Augustin et la métaphysique contemporaine}

I. Dépsychologiser la lecture de saint Augustin

Quand saint Augustin a recours, faute de mieux, au terme de personne pour tenter de dire le mystère de la Trinité plutôt que de rester sans rien dire, ce terme a déjà une longue histoire, assez chaotique, et qui se trouve traversée par des tensions très fortes entre prosôpon et persona, ou encore entre le masque et le visage, ou enfin entre sa signification juridique première et sa signification biblique naissantel. De fait, la signification du terme de personne n'a cessée de varier et il est très difficile de mettre en évidence dans cette évolution une idée téléologique s'affirmant peu à peu dans ce devenir de façon intentionnelle ou de façon inintentionnelle, sauf à privilégier arbitrairement une définition de la personne et à relire toute l'histoire de la métaphysique à partir du prisme de cette définition. En outre, il semble bien qu'il n'y ait pas une histoire de la personne, mais au moins deux histoires de la personne: l'histoire de sa signification juridique et l'histoire de sa signification biblique qui est une signification ontologique. Or ces deux histoires apparaissent comme irréductibles l'une à l'autre et, de ce fait, l'existence même d'un véritable "concept" de personne est mise en cause, puisque posséder deux définitions c'est ne pas avoir de définition. Pour la première histoire, la plus souvent écrite ${ }^{2}$, qui va des Stoïciens à Locke, Kant et Hegel, il s'agit de distinguer les personnes et les choses d'un point de vue juridique: une personne a des droits et des devoirs et elle répond d'elle-même. En ce qui concerne la seconde histoire, qui débute avec les controverses christologiques et trinitaires, et plus précisément avec saint Augustin, comme on va le montrer, el-

\footnotetext{
${ }^{1}$ Sur cette première histoire du terme de personne voir le livre pénétrant de P. Cormier, Généalogie de Personne, Critérion, Paris 1994.

2 Voir, par exemple, T. Koвusch, Die Entdeckung der Person. Metaphysik der Feiheit und modernes Menschenbild, Herder, Freiburg-Basel-Wien 1993. Le présent article se propose de mettre en évidence une tout autre invention de la personne.
} 
le s'accomplit avec saint Thomas d'Aquin, et semble être suspendue jusqu'à la phénoménologie contemporaine qui lui offre une deuxième naissance. Cette fois le terme de personne n'a pas d'abord une signification anthropologique, mais s'impose à partir d'une interrogation exégétique, et c'est pourquoi il s'agit de mettre en évidence la dignité absolue de la personne, incomparable avec la chose, et sa nature événementielle et dialogique. En ce qui concerne ces deux histoires, il est hors de question de s'en tenir à une opposition simpliste entre un concept théologique et un concept philosophique de personne car le terme de personne reçoit un usage anthropologique et un usage théologique dans les deux cas. L'idée d'un concept purement anthropologique de personne, sans aucun horizon théologique, est une idée très récente, même si certains estiment qu'elle est en puissance dès la découverte de la personne. En outre, ces deux histoires se sont entrelacées et elles affirment toutes les deux la nécessité de distinguer la personne et la simple réalité naturelle. Elles se rejoignent donc autour de l'idée simple que la personne n'est pas une chose, mais pour se séparer immédiatement sur la signification de cette distinction ontologique. D'un côté l'être-personne est compris comme ce qui s'ajoute à notre être-chose et permet de dire plus précisément ce que nous sommes à partir de l'esprit ou de la conscience, d'un autre côté l'être-personne demeure compris comme une totalité indivisible, âme et corps, qui ne partage rien avec le simple être-là-devant de la chose.

Cette rapide mise en perspective de la tension, voir de la division, qui traverse l'histoire du terme de personne permet au moins de souligner à quel point l'usage qui est fait aujourd'hui du terme de personne est vague et indéterminé et en outre ignorant de l'histoire à laquelle il appartient. Or, c'est ce caractère bien connu, allant de soi (la personne est l'homme en tant que conscient de luimême), de la représentation moderne de la personne qu'il s'agit d'interroger, et sans doute de déconstruire, afin que la personne redevienne pour elle-même une question, avant de se demander quelle place au soleil elle est capable de se préserver. En effet, dans un style husserlien, il est possible de comprendre l'indétermination actuelle du concept de personne non comme le résultat d'une inculture de l'humanité, mais comme la conséquence d'une certaine définition de la personne. La méditation historique du dernier Husserl enseigne au moins que l'errance de son monde le philosophe doit se l'attribuer, doit la comprendre comme l'erreur d'un certain rationalisme et qu'il doit y répondre philosophiquement en redonnant aux concepts leur signification authentique. Autrement dit, la crise actuelle de la personne ne tient-elle pas d'abord à une certaine philosophie de la personne qui a conduit à une conception très abstraite? En effet, avec la compréhension moderne de la personne, qui s'affirme avec Locke et se déploie jusque dans les dernières ramifications du personnalisme, la personne se comprend comme n'étant pas une simple chose parce qu'elle est consciente d'elle- 
même, parce qu'elle répond de ses actes et est ainsi la cause d'elle-même, la créatrice de son être. La capacité a priori de prendre conscience de soi qui définit la subjectivité moderne apparaît comme le pouvoir originaire à partir duquel il est possible de se "produire" soi-même sans fuir le monde, mais en transformant tout ce qui arrive en élément de sa propre genèse. Comme le montre Husserl dans la quatrième des Méditations cartésiennes, la constitution de la transcendance du monde s'identifie à l'auto-constitution de l'ego transcendantal. Tout sens, y compris le sens "objet transcendant" est ce qui se constitue pour une conscience et toute transcendance constituée est une transcendance dans l'immanence, c'est-à-dire le résultat d'une validation d'être, d'une effectuation de conscience. Certes, le sens n'est en rien projeté de l'extérieur sur le monde, mais tout objet, un arbre, un livre, ma chair, l'autre homme, un cercle, Dieu luimême, est un noème constitué. Dans cette métaphysique de la subjectivité il n'y a pas de monde sans conscience et pas de conscience sans monde et l'auto-explicitation de l'ego, qui est le chemin cartésien, est-ce qui permet de répondre à la question “qu'est-ce que l'étant?". Tout ce qui est, et même ce qui viendrait me surprendre, me blesser ou me convoquer, demeure un sens qui s'annonce dans la conscience. Avec cette activité de l'ego il n'en va pas uniquement de la possibilité d'atteindre l'objet, mais aussi de la possibilité même de l'objectivité: l'égologie transcendantale est l'ontologie fondamentale. L'être absolu de l'ego n’est pas une île, comme il est dit au $§ 41$, mais ce qui est la source de toute identité: la constance de l'ego dans sa propre genèse est l'origine de la constance de l'étant, étant qui est en fait l'idée d'un infini travail théorique. Ainsi, le radicalisme de l'égologie transcendantale développé par Husserl peut être compris comme l'une des expressions les plus achevées du personnalisme contemporain dans lequel la personne est vraiment comprise comme acte en s'affranchissant de tout naturalisme et de tout psychologisme qui conduisent toujours à faire retomber la personne au rang de simple chose. Nul mieux que Husserl n'a mis en évidence l'abîme toujours persistant du naturalisme, d'une compréhension zoologique de la personne, qui est dominante dans les sciences humaines et très efficace dans la société contemporaine ne serait-ce que dans le fait de se demander si certaines personnes âgées, ou handicapées, ou malades, ont vraiment une légitimité à être. Le simple fait que la question soit posée atteste d'une compréhension de la personne comme nature, en incluant dans ce naturalisme spontané et dominant tout ce qui relève de la nature sociale ou psychologique: la personne serait une réalité naturelle qui ne se différencie de la simple chose physique que par une couche complémentaire d'humanité faite de caractères psychiques. Contre un tel naturalisme, le personnalisme phénoménologique montre que prendre conscience de soi c'est découvrir et ne cesser de découvrir en soi l'ego transcendantal afin de pouvoir se vouloir absolument soi-même. 
Il était nécessaire de convoquer, très brièvement certes, cette forme achevée de la métaphysique de la subjectivité qu'est l'idéalisme transcendantal et égologique husserlien pour engager une interrogation sur le concept de personne et une interrogation sur saint Augustin. Une question, donc, sur la personne en premier lieu: suffit-il de s'affranchir de tout naturalisme, de séparer radicalement chose et personne, pour abandonner toute perspective ontique selon laquelle la personne demeure un étant qui possède la réflexion, qui se constitue lui-même dans l'unité d'une histoire et qui peut vivre dans une responsabilité absolue? Dans cette philosophie de la subjectivité, la personne demeure un "sujet" d'actes intentionnels, et on peut se demander, bien évidemment à partir de Heidegger, si cela permet d'avoir accès au sens d'être de la personne et même si ce sens d'être est véritablement interrogé ou bien s'il n'est pas encore présupposé comme allant de soi. Le radicalisme de l'égologie transcendantale ne suffit peut-être pas à l'élucidation de la séparation ontologique de la chose et de la personne et il est nécessaire de remettre en cause cette compréhension de la personne comme toujours déjà auprès d'elle-même, comme auto-constituante, et comme auto-législatrice. Une interrogation sur saint Augustin, ensuite, dans la mesure où il est souvent considéré, à tort, comme l'inventeur du moi moderne et, de ce point de vue, Husserl lui-même estime que l'ego transcendantal accomplit la vérité du Noli foras ire, in te redi, in interiore homine habitat veritas cité plusieurs fois et notamment comme ultime phrase des Médiations cartésiennes. Même dans les commentaires les plus contemporains, saint Augustin est compris comme un penseur de la subjectivité, de la réflexivité de la conscience, et le retour en soi est compris comme un retour à soi au sens d'une introspection ou d'un examen de conscience ${ }^{3}$. Ce somnambulisme du commentaire, qui consiste d'une part à rendre saint Augustin respectable ou présentable aujourd'hui en le "dé-théologisant" ou, au moins, en isolant quelques textes de pure philosophie dans un océan théologique, et, d'autre part, à l'abstraire de son historicité propre, conduit

3 Il est inutile d'identifier tous les lieux d'une telle "non lecture" de saint Augustin, mais il est possible de citer au moins le livre de C. TAYLOR au titre grandiloquent Sources of the self, trad. franç. Les sources du moi par C. Melançon, Seuil, Paris 1998, notamment le chapitre 7 In interiore homine et par exemple p. 178: «Le virage augustinien vers le moi était un virage vers la réflexivité radicale, et c'est ce qui a rendu irrésistible le langage de l'intériorité. La lumière intérieure est celle qui brille dans notre présence à nous-mêmes, inséparable du fait que nous sommes des créatures dotées d'un point de vus subjectif. Ce qui la distingue de la lumière extérieure est justement ce qui rend l'image de l'intériorité si forte, parce qu'elle illumine l'espace dans lequel je suis présent à moi-même». Il ne s'agit même pas d'une interprétation possible de saint Augustin, mais vraiment d'une absence d'écoute de cette pensée de l'écoute et cela à partir d'un concept soi-disant moderne d'intériorité. Il y a certes une pensée de l'intériorité dans l'œuvre augustinienne, mais elle est justement d'une tout autre nature et procède d'une tout autre source que l'intériorité issue de la réflexivité d'un sujet. 
à ce phantasme qui a la vie dure d'un saint Augustin pré-cartésien, pré-kantien et pré-husserlien ${ }^{4}$. Or une telle désinvolture dans l'interprétation critique, son caractère aussi généralisé, ne tient pas simplement au fait qu'on lit le passé à partir des questions du présent, mais tient à un psychologisme ambiant qui ne rend justice ni aux philosophies de la subjectivité, ni à saint Augustin. En effet, la transformation de saint Augustin en champion du regard intérieur tient fondamentalement à une indigence conceptuelle, excusable dans les lectures naïves ou spontanées, moins compréhensible dans les études historiques, qui consiste à confondre la personne et l'ego, qui consiste à les tenir pour synonymes dans l'indétermination totale du terme d'intériorité portant à lui seul le poids du psychologisme. De fait, le terme d'intériorité semble être la source de toutes les confusions, puisqu'il conduit à faire de Husserl et de saint Augustin deux philosophes de l'intériorité, alors qu'en réalité ni l'un ni l'autre ne sont des penseurs d'une intériorité s'opposant à une extériorité. Le terme d'intériorité nivelle les différences au point de réunir saint Augustin et Husserl dans une lecture subjectiviste non seulement anachronique, mais surtout fausse. Saint Augustin n'a pas introduit l'intériorité de la réflexivité radicale et justement l'intériorité exposée qui est décrite dans les Confessions ne se définit pas d'abord par la réflexivité. Ce n'est qu'en se débarrassant de ce préjugé tenace, lié à un naturalisme inaperçu, qui consiste à voir en saint Augustin un philosophe de la subjectivité, de la conscience de soi, de la réflexivité radicale, qu'il est possible de comprendre en quoi avec lui s'effectue une invention de la personne qui n'est précisément pas une invention de l'ego. Si pour saint Augustin l'âme est bien "mon" âme, ce caractère mien ne doit pas être référé à un étant intramondain et qui se caractérise par la réflexion. Cette figure de la personne ne correspond ni à saint Augustin, ni à Husserl, et manque à la fois l'ipséité par grâce que l'ipséité issue de l'égoïté. Saint Augustin peut se demander «quoi de plus proche de moi que moi-même?» Quid autem proprinquius me ipso mihi? ${ }^{5}$, il est essentiel de résister à la tentation de faire du pronom réfléchi, du "me", un substantif. En effet, cette question sur soi ne peut pas être lue comme issue d'une objectivation de soi, comme issue de l'acte d'un sujet qui devient pour lui-même un objet, comme dans les philosophies de la subjectivité pour lesquelles la cons-

\footnotetext{
${ }^{4}$ Le plus grave est que ce somnambulisme semble se poursuivre en dépit des mises en garde déjà anciennes notamment de Goulven Madec et d'Isabelle Bochet qui montrent en quoi l'intériorité augustinienne n'a rien de commun avec l'intériorité husserlienne, par exemple, et même s'y oppose radicalement. Voir, entre autres, de G. Madec, Petites études augustiniennes, Institut d'Etudes Augustiniennes, Paris 1994, et d'I. Bоснет, Le firmament de l'Ecriture. L'herméneutique augustinienne, Institut d'Etudes Augustiniennes, Paris 2004.

${ }^{5}$ Confessions X, xvI, 25, Etudes Augustiniennes, Paris 1992 («Bibliothèque Augustinienne», 14), pp. 184-185.
} 
cience s'objective elle-même dans une conscience de soi qui devient une conscience du soi. On comprend alors que la lecture qui consiste à substantiver le "me" résulte de la définition implicite de la personne par la conscience de soi, c'est-à-dire comme ce qui se regarde soi-même. Or la conscientia pour saint Augustin est l'organe de l'âme au sens où elle est cette capacité de voir qui n'est pas d'abord une capacité de se voir. Autrement dit, la conscientia n'est pas le lieu d'un dédoublement de soi, d'une Ichspaltung, mais elle est le lieu où Dieu parle, où Dieu “me" parle, et c'est pourquoi la bonne conscience est celle qui est ordonnée à la parole de Dieu6. L'unique objet des Confessions, celui à partir duquel les textes deviennent intelligibles, c'est le Christ, et c'est pourquoi les Confessions ne sont pas une introspection, ne sont pas une méditation sur le moi ou sur le soi. En effet, saint Augustin ne cesse de montrer que l'acte de la confession ne conduit pas à un moi, mais conduit à Dieu en soi devant lequel je peux répondre "me voici". En conséquence, mon être n'est pas ici objectivé dans une réflexion, il n'est pas ce qui est produit par une pensée qui se dit en première personne, mais il est l'objet de l'amour de Dieu. Ainsi, le point de départ nécessaire d'une lecture de saint Augustin c'est que le "me" ne doit surtout pas être interprété à partir du "je me pense" et du "je me veux" des philosophies de la réflexion. En effet, être vrai ou être soi ce ne sera justement pas se vouloir et se penser, mais ce sera, dans l'amour, être contraint à son expérience propre. Enfin, on peut se demander à quelle philosophie correspond cette figure radicale que serait une ipséité comprise comme une intériorité close et donnée à elle-même dans une auto-affection et dans l'exclusion de toute transcendance. Une telle conception de l'ego n'appartient pas à Descartes ${ }^{7}$ ou à Husserl: pour l'un comme pour l'autre la donnée à soi est inséparable de la relation à l'objet ${ }^{8}$. Telle est l'intentionnalité de la conscience en dehors de laquelle l'ego n'est qu'une forme vide et non un être qui s'éprouve lui-même dans la connaissance du monde. Dès lors, la question en retour sur l'intériorité augustinienne qui la donne à voir comme n'étant pas un substantif mais un acte de parole permet de mieux comprendre le souci de la phénoménologie contemporaine de désubstantifier, dépsychologiser, la subjectivité, mais permet également de comprendre que l'ipséité augustinienne est par principe tout autre qu'une ipséité issue d'une métaphysique de la subjectivité et que c'est pour cela qu'elle peut donner un avenir à la réflexion sur la personne au-delà de l'idée du sujet. De fait, la philosophie du su-

\footnotetext{
6 Voir C. MaYer, Augustinus Lexikon, article Conscientia, vol. I, Schwabe, Basel 1986, coll. 12181228.

7 Voir G. OLivo, Descartes et l'essence de la vérité, Puf, Paris 2005 («Epiméthée», 1), p. 333.

${ }^{8}$ Qu'il me soit permis de renvoyer ici à mon livre Husserl et l'énigme du monde, Seuil, Paris 2000.
} 
jet n'a pas pu reprendre le concept biblique de personne et s'en est toujours tenu au concept juridique 9 .

Certes, saint Augustin n'est pas le premier à avoir recours au terme de personne et il hérite de l'histoire déjà fort complexe de ce mot, néanmoins il est possible de soutenir que saint Augustin a "inventé" le concept de personne pour au moins deux raisons: d'une part, le mot personne n'était pas encore bien fixé et pouvait encore signifier le rôle ou la place à tenir et il revient à saint Augustin d'avoir déterminé le sens de l'expression una persona pour dire que le Christ total est une seule voix et une seule chair. D'autre part, toute la réflexion trinitaire de saint Augustin lui permet au livre VII du De Trinitate d'investir ce terme d'une signification nouvelle à partir d'un usage exégétique. Ce sont les besoins de la controverse qui conduisent à avoir recours au terme de personne et à lui donner une signification événementielle et relationnelle pour qu'il soit une alternative au terme de substance. Il s'agit bien de dire une figure de l'ipséité inconnue de la pensée grecque et méconnue de la pensée moderne qui psychologise saint Augustin. Dès lors, ressaisir aujourd'hui la nouveauté du concept augustinien de personne permet de comprendre comment un thème chrétien s'introduit au cœur de la métaphysique et loin de la dénaturer peut lui redonner un avenir en libérant du concept moderne de personne introduit par Locke, fixé par Kant et critiqué par Hegel, et qui est avant tout un concept juridique de personne même s'il prend aussi une signification éthique ${ }^{10}$. La pensée de l'identité personnelle, depuis Locke, qui cherche à comprendre ce que je suis à partir de ce que je fus, et cela jusque dans sa figure la plus actuelle qu'est la théorie de l'identité narrative développée par Paul Ricoeur, s'oppose irréductiblement à la thèse augustinienne selon laquelle ce qui est mien ne peut se comprendre qu'à partir de ce qui me donne d'être, de ce qui m'appelle. Il n'y a pas d'accord possible entre l'ipséité transcendantale, même dans sa figure la plus ouverte représentée par Ricoeur, et l'ipséité responsive de saint Augustin. De ce point de vue, le concept augustinien de personne peut être encore aujourd'hui l'avenir de la métaphysique en ce qu'il signifie un mode de l'existence qui ne se trouve ni

\footnotetext{
9 Voir J.-L. Chrétien, Saint Augustin et les actes de parole, Puf, Paris 2002 («Epiméthée»), p. 241 : «Quoi de plus évangélique que d'affirmer que c'est là où je me perds et m'oublie, là où je ne cherche pas à sauvegarder ce qui était mien par avance, que je deviens proprement moi-même, c'est-à-dire que je m'approche de mon éternelle vocation? Cela vaut aussi de la parole. Dans cette pensée de la mienneté, il n'y va pas d'une préfiguration encore confuse des philosophies modernes de la subjectivité, mais d'un mouvement provenant d'une autre source et d'une autre lumière, et qui a force de mission pour survivre au désastre de ces dernières».

10 Voir G.W.F. Hegel, Principes de la philosophie du droit § 36, trad. franç. R. Derathé, Vrin, Paris 1975, p. 97: «La personnalité contient, d’une manière générale, la capacité du droit. Elle constitue le concept et le fondement - lui-même abstrait du droit abstrait ou formel. Par suite, l'impératif du droit sera: sois une personne et respecte les autres comme personne».
} 
dans le je transcendantal husserlien, ni dans le Dasein heideggérien. Est-ce saint Augustin qui est pré-phénoménologue ou n'est-ce pas la phénoménologie qui, en rompant de plus en plus avec son propre naturalisme, se découvre toujours davantage comme pré-augustinienne?

Comme on va le voir, la personne selon saint Augustin est un acte de parole qui consiste à répondre à la question "qui parle?", "qui est-il?" et cette Werheit, pour parler avec Heidegger, n'est accessible qu'à celui qui est capable de renoncer à la revendication d'être l'origine de son ipséité et qui accepte de se recevoir de ce qui le touche. Le long travail de la confession ne part pas de la question "qui suis-je?", mais il est ce qui permet peu à peu d'entendre la force et la signification de cette question. La personne n'est pas l'être qui se trouverait d'emblée luimême dans un acte de réflexion comme pouvoir constant de dire je, mais l'être qui fait d'abord l'épreuve de son obscurité et de son inconstance, autrement dit, l'être qui fait d'abord l'expérience de son néant. Dès lors, si saint Augustin avec l'invention du concept de personne décrit une constance, il ne s'agit ni de près, ni de loin, de la constance d'un étant doué de réflexion, d'une auto-constance, mais bien au contraire d'une constance qui vient du fait d'être renouvelé par une altérité. Il n'y a de véritable constance qu'à se laisser transformer par la vérité. Des méditations cartésiennes se définissent comme une auto-élucidation du sujet connaissant, mais des médiations augustiniennes répondent à un tout autre projet comme Heidegger l'avait déjà vu: «La certitude de soi (Selbstgewissheit) et le se posséder (sich-selbst-Haben) au sens d'Augustin sont quelque chose de totalement différent de l'évidence cartésienne du cogito» ${ }^{11}$.

\section{L'essence événementielle de la personne}

Contrairement à ce qu'écrivait Husserl à la fin des Médiations cartésiennes, saint Augustin n'est pas une simple étape, même importante, dans la mise en évidence progressive de la signification transcendantale du "connais-toi toi-même" dans la mesure où le principe premier est le "transcende-toi toi-même"12 qui doit être compris comme un devenir autre radical. Pour le dire d'une façon un peu familière, être soi consiste, selon l'expression française, à "aller voir ailleurs si j'y suis", non pas pour retrouver ce que l'on est déjà, mais pour découvrir ce que l'on n'est pas encore. Dans cet acte de se transcender se joue la possibilité d'être soi ou de se perdre. Dès lors, le fameux "retour en soi" n'est en rien un re-

11 M. Heidegger, Phänomenologie des religiösen Lebens, Klostermann, Frankfurt 1995 («Gesamtausgabe», 60) p. 298.

12 Voir La vrai religion, XXXI, 72 («Bibliothèque Augustinienne», 8), p. 130. 
tour à soi, ou le retour à quelque chose comme un "moi”, mais précisément l'acte d'aller au-delà de soi-même pour écouter la vérité. C'est une telle écoute, dans sa constance relative et finie, qui donne à l'existence humaine sa tenue ${ }^{13}$. La personne humaine n'est qu'en réponse et n'est elle-même qu'aussi longtemps qu'elle écoute la vérité. Or dans cette écoute elle trouve sa loi car écouter la vérité sans lui obéir ce n'est pas vraiment l'écouter, c'est lui demeurer toujours extérieur. L'appel de Dieu envoie l'homme vers lui-même en l'envoyant au-delà de lui-même et c'est précisément un tel devenir soi qui ne peut pas être pensé à partir de la problématique de la conscience de soi. Il ne s'agit en aucun cas ici d'un sujet qui s'appelle lui-même, même du plus loin et du plus haut que luimême, mais véritablement de l'appel d'une altérité qui dans cet appel donne à l'homme sa forme véritable ${ }^{14}$. Ainsi, dans le retour au plus intime d'elle-même l'âme humaine découvre l'excès de ce qui l'habite et qui n'est justement pas “à elle" dans la mesure où elle comprend tout au contraire que c'est elle qui appartient à la vérité. Contre la lecture subjectiviste de la doctrine du maître intérieur, il est nécessaire d'insister sur le fait que l'âme dans le retour sur elle-même ne découvre surtout pas un "moi", mais une vérité qui la transforme et à laquelle elle peut répondre le "voici, je viens" des Psaumes. Dès lors, cet appel de Dieu n'est pas un simple événement que j'aurais à m'approprier, il n'est pas l'occasion d'un rapport de soi à soi, mais il est l'unique source du devenir soi. De ce point de vue, le Christ n'est pas réductible à un idéal humain, il n'est pas l'homme idéal d'une catharsis, mais il est l'être devant lequel je me tiens dans la parole de la confession qui est une parole de conversion. Tout homme a la possibilité de devenir lui-même devant le Christ et la confession est alors la plus haute possibilité de l'existence humaine dans la mesure où elle est le lieu où l'homme est à l'écoute de la vérité. Saint Augustin décrit ainsi une ipséité responsive issue du regard vers l'Etre «dans l'éclair d'un coup d'œil frémissant» ${ }^{15}$. L'Etre, qui est aussi ici la personne du Christ, est ce qui m'appelle et me transforme, il est ce qui me reconduit à moi-même et à ce qu'il m'est donné d'avoir à être. Le livre X des Confessions se termine par l'appel au Christ médiateur et il ne s'agit en aucun cas d'un appendice théologique d'un texte philosophique sur la mémoire, mais du souci de rappeler que le Christ est l'unique source de l'ipséité humaine. Il ne s'agit pas non plus d'une méditation sur Dieu cause de soi ou

13 Voir Chrétien, Saint Augustin et les actes de parole cit., p. 25: «Nous ne pouvons tenir, nous tenir, nous tenir debout qu'à écouter».

14 Voir Bochet, Le firmament de l'Ecriture. L'herméneutique augustinienne, Institut d'Etudes Augustiniennes, Paris 2004, p. 316: «Le sujet, dans la perspective d'Augustin, est donné à lui-même par Dieu, qui peut, seul, le mener à sa forme véritable, et que ce don fait par Dieu de toute éternité s'expérimente dans le concret de l'histoire».

15 Confessions VII, XVII, 23 («Bibliothèque Augustinienne», 13), p. 629. 
sur l'autosuffisance de l'essence divine, mais de la volonté de montrer que tout être soi trouve sa seule source dans un être qui est l'être-même parce que tout son être est de se donner.

La mémoire n'est pas dans les Confessions le lieu privilégié de la constitution de soi, alors qu'elle l'est dans la plupart des philosophies de l'identité personnelle. La mémoire n'est pas le lieu où je me retrouve moi-même, à la fois sujet et objet, mais le lieu où résonne une autre voix que la mienne dont l'écoute permet de me tenir dans le déploiement du temps. Sans étudier ici les différents textes sur la mémoire ${ }^{16}$, il suffit d'indiquer qu'ils culminent dans la question de la mémoire de Dieu. Que cette mémoire de Dieu soit la source unique de notre être vrai confirme que saint Augustin est ici aux antipodes d'une conception idéaliste du sujet dans la mesure où il ne pense pas l'ipséité humaine comme une production du je, de son pouvoir de synthèse, et où il ne décrit pas la mémoire de Dieu comme la présence d'une Idée constitutive de la subjectivité. Dieu n'est pas pour saint Augustin une Idée mais une personne et c'est pourquoi la mémoire de Dieu n'est donc justement pas dans la mémoire comme ce qu'elle pourrait contenir: Dieu n'est pas trouvé en l'homme mais en lui et c'est pourquoi toute la constance issue de la mémoire vient de l'histoire de Dieu en soi. La mémoire de Dieu n'est donc pas le résultat d'une effectuation de conscience, mais elle est ce mouvement de transcendance en lequel consiste notre ipséité. L'homme ne se rassemble que dans l'unique désir d'être la voix de son origine et déjà ce mouvement de transcendance est rendu possible par le Christ qui permet que «oubliant le passé, tourné non pas vers les choses futures et transitoires, mais vers celles qui sont en avant et vers lesquelles je suis non pas distendu mais tendu, je poursuis, dans un effort non pas de distension mais d'intention, mon chemin vers la palme à laquelle je suis appelé là-haut»17. En dépit de la présence dans ce texte d'une des rares occurrences d'intentio dans les Confessions, il ne s'agit pas d'une pensée de l'intentionnalité et la personne humaine n'est donc en rien l'être qui se produit lui-même, mais elle est, tout au contraire, cette âme mendiante qui accepte d'être renouvelée de l'intérieur par ce qui vient du Christ et ne peut venir que de lui. Il n'y donc rien de commun entre la subjectivité pleine d'elle-même, pleine de sa propre activité de constitution, de l'idéalisme husserlien et cette âme mendiante qui en confessant son injustice laisse la vérité la transformer, lui donner une forme. Tout le projet des Confessions est bien de montrer que l'homme ne tire pas de lui-même sa lumière pro-

16 Voir mon article Mémoire de soi et épreuve de l'altérité selon le livre X des Confessions de saint Augustin, in C. DorniER (éd.), Ecriture et exercice de la pensée, Presses Universitaires de Caen, Caen 2001, pp. 49-73.

17 Confessions XI, XXIX, 39 («Bibliothèque Augustinienne», 14), p. 339. 
pre et ne peut se découvrir lui-même qu'en se laissant éclairer par une lumière qui vient d'ailleurs, qui vient de l'être même. On voit alors combien il est absurde de vouloir séparer dans les textes de saint Augustin des pépites philosophiques de résidus théologiques, car c'est bien à partir de cette non séparation que saint Augustin peut décrire ces dimensions événementielle et responsive de la personne, qui ne devient elle-même qu'à partir de l'événement d'une rencontre avec Dieu; c'est en effet dans cette rencontre que l'existence personnelle est à la fois déconstruite et reconstruite. Autrement dit, c'est dans un même mouvement que la personne découvre son obscurité et qu'elle découvre la lumière qui la fait advenir à elle-même. Il faut donc encore une fois résister à la tentation de substantiver le soi, car être soi c'est être donné au sens d'être envoyé pour transmettre ce que l'on a reçu: on n'est une personne qu'à se faire signe du Christ.

Dans cette perspective il y a un lien eidétique entre la personne et l'amour que les philosophies de la subjectivité peuvent parfois reconnaître mais auquel elles ne peuvent donner aucune place véritable. La dualité de la raison et du sentiment relève elle-même de la problématique du je pur et ce psychologisme interdit de comprendre en quoi l'amour est le mouvement de transcendance propre à l'existence humaine. Il est ainsi nécessaire de se libérer de la représentation de l'amour comme de ce qui s'éprouve dans l'immanence de l'intériorité close pour retrouver l'idée augustinienne que dans l'amour je suis à la fois au plus intime de moi-même et hors de moi-même. L'amour est décrit par saint Augustin non pas comme la propriété d'un étant doué de réflexion, mais, ontologiquement, comme le mode d'être hors de soi dans lequel un "soi" s'éveille irréductible à toutes les productions propres de l'homme. Ce qui aime en soi ce n'est pas quelque chose comme "le moi", ce n'est pas la capacité à se donner soi-même l'impératif d'être une personne et, ensuite, de respecter l'autre comme une personne, mais c'est un pouvoir être qui ne m'appartient pas et qui pourtant m'individue. En conséquence, l'amour n'est pas ce qui vient s'ajouter à mon être, il n'est pas cette couche de spiritualité qui vient s'ajouter à une couche naturelle et à une couche psychique, mais il est mon être même. Certes, il n'y a pas univocité de la personne en l'homme et en Dieu car en nous l'amour n'est pas la personne même, mais il est bien ce qui nous donne notre forme véritable. Au sommet du livre XIII des Confessions saint Augustin écrit le célèbre pondus meum, amor meus selon lequel l'amour est un poids qui emporte l'âme vers son lieu propre ${ }^{18}$. La patience de l'amour consiste ainsi à renoncer à s'élever par soimême, à se porter vers une fin que l'on se serait fixé par soi seul, pour laisser

18 Sur l'identification d'amor à pondus voir aussi Cité de Dieu XI, XXvIII; Lettre 55, 10,18; De la musique VII, 11, 29; De Trinitate VI, x, 12. 
l'amour progresser en soi. Dans cette transcendance dans la passivité, qui n'est pas sans une certaine activité, s'effectue un retournement complet de notre être et une mutation spirituelle par lesquels nous devenons tout autre que ce que nous nous proposions d'être par nous-mêmes. En décrivant l'amour comme poids saint Augustin est aux antipodes de la subjectivisation moderne de l'amour qui conduit à la scission entre l'amour pathologique et l'amour pratique. Le poids comme mouvement spirituel est la réalité même de l'amour et avec cette idée de poids il est possible de comprendre en quoi l'amour est à la fois un mouvement de transcendance et une force immanente à notre être. La possibilité du rassemblement de soi, d'une constance propre à l'homme, vient alors de la capacité à ne pas faire obstacle à cette force pour être tout entier dans son mouvement de conversion. A l'opposé de l'idée d'un sujet autolégislateur et autotélique, l'amour se dévoile comme étant au plus intime de la personne un poids subi qui cependant la libère d'elle-même, de son auto-idolâtrie, pour la rendre à sa vocation. Il n'y a pas ici à choisir entre l'amour de soi et l'amour de la vérité dans la mesure où consentir à ce poids c'est être miséricordieux avec soi-même, c'est véritablement s'aimer en donnant à notre être ce dont il a véritablement faim et soif. L'alternative propre à l'existence humaine n'est donc pas celle de l'autonomie et de l'hétéronomie, mais celle du poids de la chair et du poids de l'amour. De même que les corps ne sont pas pensables sans leur poids, la personne humaine n'est pas pensable sans l'amour qui la fait être là où elle peut se rassembler elle-même.

III. L'essence relationnelle de la personne

Jusqu’à maintenant le terme de personne a été utilisé par anticipation dans la mesure où c'est un terme qui appartient d'abord chez saint Augustin à l'exégèse prosopographique qui consiste à déterminer qui parle dans l'Ecriture. Si la personne est une «réalité singulière et individuelle»19, il s'agit bien de répondre à la question "quelle personne parle?"20. Pour comprendre que le Christ puisse parler comme personne corporelle sans devenir deux personnes, il est nécessaire de dissocier la notion de personne de l'idée de sujet subsistant pour la concevoir comme la manifestation par la voix d'un être en relation. A partir de là il est possible de soutenir que saint Augustin "invente" la personne, même si

19 De Trinitate, VII, vI, 11 («Bibliothèque Augustinienne», 15), p. 543.

20 Sur l'exégèse prosopographique et sur la place de saint Augustin dans l'expression una persona voir M.-J. Rondeau, Les commentaires patristiques du Psautier, Pont. Inst. Studiorum Orientalium, Roma 1985 («Orientalia Christiana Analecta»). 
c'est d'une façon tout à fait hésitante, dans la mesure où l'essence relationnelle de la personne n'est plus seulement l'une des significations possibles mais devient sa signification principale. Dès lors, sans poser en principe l'univocité de la personne humaine et de la Personne divine, il faut reconnaître que c'est à partir du mystère de la Trinité que l'essence de la personne peut être vue. Pour saint Augustin, l'usage latin de parler d'une essence et de trois personnes à propos de Dieu consiste surtout à ne pas rester sans rien dire ${ }^{21}$. Il s'agit d'une façon commode de parler qui ne doit pas faire oublier que la Trinité déborde les ressources du vocabulaire humain. En outre le terme de personne n'est pas réservé à Dieu: “Personne" est un terme générique, si bien qu'on peut l'appliquer à l'homme aussi, alors qu'il y a tellement loin de l'homme à Dieu»22. L'homme est une personne comme image de la Trinité et c'est donc à partir de l'Idipsum qui est pourtant trois personnes qu'il est possible d'avoir accès à l'ipséité de la personne humaine, sans pour autant poser une communauté d'essence entre l'homme et Dieu.

Ainsi, les controverses théologiques ont en quelque sorte "forcé" saint Augustin à user du terme de personne pour souligner que le Père, le Fils et l'Esprit ne sont pas seulement des noms différents pour une même essence, mais se distinguent réellement l'un de l'autre. Mais dans le même temps il conserve au terme de personne sa signification absolue et, de ce point de vue, il n'y a pas trois dieux mais une seule personne. Les commentateurs ont souvent souligné cette ouverture de saint Augustin vers la signification relationnelle de la personne, notamment quand il compare la Trinité à la relation de trois amis, et son souci de ne pas perdre l'unité de Dieu. Cette hésitation de saint Augustin permet de souligner qu'il ne s'agit pas simplement de passer de la substance à la relation comme l'a supposé un peu naïvement un certain personnalisme dialogique. Ici, quand saint Augustin parle "des personnes", personne signifie la singularité, et quand il parle de "la personne", personne signifie la substance. Le Père, le Fils et le Saint Esprit n'étant ni trois noms, ni trois essences, le terme de personne semble être le seul qui puisse dire en même temps la singularité des personnes divines et leur profonde identité. L'invention de la personne correspond exactement à cela: la reconnaissance d'une singularité qui n'est pas accidentelle et qui introduit une distinction mais pas une séparation. La radicale nouveauté de ce concept de personne par rapport à la pensée grecque tient à cette idée que la personnalité est une relation essentielle qui est une relation d'amour. Même si l'homme n'est pas seulement une personne, en lui l'unité de personne est radi-

21 Voir De Trinitate, V, Ix, 10 («Bibliothèque Augustinienne», 15), p. 449.

22 De Trinitate, VII, IV, 7 («Bibliothèque Augustinienne», 15), p. 533. 
calement autre que l'unité de nature et en outre elle ne vient pas seulement s'ajouter à une unité qui la précéderait. La paternité et la filiation introduisent cette idée neuve que l'on est soi par sa relation à une altérité. En Dieu tout ne peut pas se dire selon la substance, mais, d'une certaine façon, c'est aussi vrai en l'homme. Certes, Dieu est l'Etre-même, il est celui qui se tient lui-même, mais en donnant à comprendre que cette tenue n'exclut pas la relation, cela donne aussi à comprendre qu'en l'homme la tenue suppose la relation à d'autres personnes. Saint Augustin ne réconcilie cependant pas pleinement relation et substance, et, de ce point de vue, il ne fait qu'anticiper la relation subsistante de saint Thomas d'Aquin, mais il n'en demeure pas moins que la personne se donne à comprendre comme un principe de distinction sans division des natures. En outre, le mystère trinitaire, en donnant à penser qu'aucune des Personnes divines ne détient la substance à elle seule, peut aussi conduire l'homme à comprendre qu'il ne peut pas être lui-même dans un acte de possession, mais seulement dans un don de lui-même à l'autre homme, c'est-à-dire dans l'amour. Dès lors l'Esprit-Saint est l'Esprit du Père et du Fils en tant qu'il est la Charité substantielle des deux et, de ce point de vue, l'Esprit Charité n'a pas l'abstraction d'une valeur et n'est pas non plus une force impersonnelle, mais il est bien une personne ${ }^{23}$. Saint Augustin "invente" la personne parce qu'il est le premier à vraiment ne plus comprendre l'homme comme un animal rationnel pour véritablement le comprendre comme image de La Trinité. Encore une fois, il ne s'agit pas simplement d'inventer un concept théologique de personne qui conduirait à juxtaposer en l'homme un concept naturel de personne et un concept chrétien de personne. En effet, ce qui se comprend à partir de l'intelligence de la Révélation peut se retrouver aussi dans le domaine naturel et avec saint Augustin c'est bien une définition ontologique de la personne qui apparaît: la singularité n'est plus comprise pour la personne comme la particularisation d'un universel, mais comme une individuation dans la relation à l'altérité. On retrouve alors le thème des Confessions: l'homme devient une personne en déployant sa capacité singulière à dire la vérité, à l'incarner, à la transmettre aux autres hommes et cet acte est sa personnalité même.

Si saint Augustin demeure prudent dans l'usage trinitaire de persona ${ }^{24}$, l'expression una persona est par contre utilisée sans réserve pour dire l'unité du

23 Voir M.-F. BERROUARD, Introduction aux homélies de saint Augustin sur l'Evangile de saint Jean, Institut d'Etudes Augustiniennes, Paris 2004, p. 214.

24 Voir M.-F. Berrouard, La défiance d'Augustin à l'égard du mot «persona» en théologie trinitaire. Notes Complementairs, in Augustinus, Homelies sur l'Evangile de Saint Jean 34-43, trad., intr. et notes par M.F. Berrouard, Études Augustiniennes, Paris 1988 («Bibliothèque Augustinienne», 73), à note 14, pp. 475-478. 
Christ $^{25}$. Il y a unité de personne dans le Christ puisque le Verbe assume la nature humaine sans confusion des deux natures. Le terme de personne ne vient donc pas signifier ici l'unité de deux rôles, mais l'unité d'un mode d'être aux hommes. La formule, «une personne, deux natures» concentre la christologie de saint Augustin: il n'y a pas de séparation en Christ entre la divinité et l'humanité et Dieu assume la nature humaine sans que le Fils devienne une nature humaine. Or la mission propre du Christ, qui est de manifester la Trinité, rend intelligible l'unité de la personne humaine. Certes en Dieu il y a identité de l'être et de la personne et non en l'homme, mais il n'en demeure pas moins qu'à partir de l'unité du Christ comme personne, l'unité de l'âme et du corps en l'homme n'est plus comprise comme accidentelle. Autrement dit, l'homme n'est pas simplement une chair à laquelle s'ajoute une âme, mais une totalité et c'est cette idée de totalité que vient signifier le terme de personne en anthropologie: c'est tout entier, âme et corps, que l'homme est à ses prochains et à Dieu, qu'il se donne et devient une personne. Parce que la personne est une totalité, elle est une relation essentielle à Dieu et aux autres hommes et c'est pourquoi sa Werheit se trouve dans son acte de se donner tout entier en réponse, tel le martyr, au don du Christ.

L'amour n'est pas ici une qualité qui s'ajoute à l'esprit ou une valeur visée par la conscience, mais il est l'esprit en acte et c'est pourquoi il est le lieu de l'ipséité, de la personnalité. C'est donc vraiment par l'amour que l'homme ne fait pas nombre avec les choses et qu'il est possible de répondre à la question "qui suis-je?". En conséquence, si être pour la personne humaine c'est être capable de connaissance et d'amour, il faut reconnaître que c'est la forme de l'amour propre au christianisme qui donne lieu à l'invention de la personne, et c'est pourquoi ce concept de personne était absent de la pensée grecque, et c'est pourquoi il est aussi absent de la métaphysique de la subjectivité: ni l'une ni l'autre ne donne une place à l'amour comme relation de personne à personne. Ainsi, la possession de soi au sens de saint Augustin n'est donc en rien une anticipation de celle du cogito, mais tient à la capacité de s'ouvrir à la vérité par l'amour. Certes, dire que l'amour est une relation de personne à personne pourrait très bien se comprendre dans une philosophie de l'intersubjectivitéé, mais il ne s'agit pas de cela pour saint Augustin qui cherche à montrer que l'amour n'est pas une capacité du moi, mais est lui-même une personne. L'amour n'est donc pas ici la

25 Voir le travail considérable de H.R. Drobner, Person-Exegese und Christologie bei Augustinus. Zur Herkunft der Formel «una persona», Brill, Leiden 1986.

26 On peut renvoyer ici à l'expression sans doute la plus conceptuelle du personnalisme français qu'est l'œuvre de Maurice Nédoncelle et à ses développements sur la genèse réciproque des consciences dans l'amour. Voir, par exemple M. NÉdoncelle, Explorations personnalistes, Aubier-Montaigne, Paris 1970 . 
possibilité d'un ego, mais c'est la personne humaine qui est une possibilité de la Charité-personne: la charité répandue dans le cœur de la personne humaine fait d'elle un témoin inébranlable du Christ. La Charité est ce qui donne à l'homme la patience de l'amour qui lui permet d'endurer le monde et de s'endurer lui-même pour dire cette vérité. Sans pouvoir développer ici l'étude de la trinité de l'âme dans le De Trinitate, on peut seulement indiquer l'impératif de ne pas psychologiser cette trinité en y voyant le rapport de trois capacités du je et la nécessité de comprendre l'amour comme un pouvoir d'union de l'esprit et de la connaissance. L'amour fait de l'existence humaine une capacité du prochain, une capacité de Dieu et donc une capacité d'être une personne. Dès lors, la personne humaine n'est pas à elle-même sa propre lumière, mais elle est l'être qui manifeste la vérité qui l'éclaire et cela jusque dans son corps. En effet, la célèbre distinction du nosse et du cogitare ${ }^{27}$ ne décrit même pas la connaissance immédiate de l'âme par elle-même comme une réflexivité originaire. Certes, toute connaissance de soi suppose une présence à soi, mais cette présence à soi ne doit précisément pas être entendue de façon réflexive comme un sujet devenant le spectateur de sa propre vie. Dans cette connaissance immédiate l'âme se connaît tout entière au sens où elle est présente à son propre acte, mais cela n’a rien d'une objectivation de soi. Il s'agit d'un se posséder soi-même dans l'ouverture même de notre esprit à la vérité à partir de laquelle l'âme ressemble à ce à quoi elle pense: «La force de l'amour est telle que les objets en lesquels l'âme s'est longtemps complu par la pensée et auxquels elle s'est agglutinée à force de souci, elle les emporte avec elle, lors même qu'elle rentre en soi, en quelque façon, pour se penser»28. C'est dire qu'il n'y a pas pour saint Augustin une cogitatio pure, une intériorité pure de toute extériorité, et que l'âme quand elle se pense, se pense toujours à partir de ce qu'elle aime. Il n'y a là rien de commun avec l'antériorité de l'a priori réflexif développé par Husserl, et quand saint Augustin soutient que même dans sa chute l'homme conserve la mémoire de lui-même, l'amour de lui-même et la connaissance de lui-même, il ne s'agit pas de la constance de la pure coïncidence d'un je avec lui-même puisque cette chute peut retirer jusqu'au pouvoir de dire je. Si l'âme conserve toujours sa structure trinitaire jusque dans sa chute, c'est que ce qui lui est donné de pouvoir être, son ipséité, ne vient pas d'elle-même, et c'est pourquoi même au plus profond de la chute le renouvellement est toujours possible. Or ce renouvellement n'a rien de comparable avec celui issu d'une auto-élucidation de l'ego par laquelle il s'éveille par lui-même à la conscience de sa tâche infinie, se ressai-

27 Voir De Trinitate, X, v, 7; XIV, vI, 8; XV, XV, 24.

${ }^{28}$ De Trinitate, X, v, 7 («Bibliothèque Augustinienne», 16), pp. 135-137. 
sit en toute certitude de la fin absolue de son vouloir, de l'Idée qui doit guider sa connaissance et son action. En effet, la mémoire de Dieu par laquelle l'homme devient lui-même selon saint Augustin est ce qui le fait devenir tout autre que ce qu'il imaginait pouvoir être par ses propres forces. Autrement dit, la conversion consiste à se comprendre selon la Charité à partir du prochain et non plus charnellement à partir des choses du monde. Certes, la personne humaine ne sera jamais comme Dieu pure relation, pur don de soi, et même dans la vision face à face elle demeurera celle qui est devenue dans une telle relation, néanmoins à chaque fois qu'elle sera la voix du Verbe elle sera parfaitement elle-même et dans cette perfection humainement possible la personne est libérée d'un achèvement indéfini vers elle-même auquel la métaphysique de la subjectivité la condamne. La personne se tient en se tenant, au moins dans la perfection d'un acte momentané, dans le Verbe et son histoire véritable est faite de ces actes dans lesquels elle est le témoin de la vérité.

\section{La personne comme réponse}

La marque même du psychologisme contemporain est d'effectuer une lecture anthropologique de tout, de saint Augustin comme de Heidegger, et de même qu'il n'est possible d'accéder à la pensée de Heidegger que par la suspension de cet anthropologisme spontané, on ne restitue à saint Augustin la vérité de son propos qu'en abandonnant toute perspective anthropologique pour lui conserver sa perspective fondamentalement théologique, ce qui ne veut pas dire non philosophique. S'il faut en finir avec cette récupération psychologiste de saint $\mathrm{Au}-$ gustin, c'est parce qu'elle interdit l'accès à cette figure de l'ipséité développée par le christianisme. Heidegger nous donne ici des yeux pour voir: sa critique radicale du personnalisme psychologisant de Dilthey, de Scheler, de Husserl et de Bergson dans Etre et temps, et notamment dans le $\S 10$, donne à comprendre que le se posséder soi-même de la personne selon saint Augustin ne doit pas être compris comme une capacité de répondre de soi a priori puisqu'elle se découvre comme toujours déjà en prise avec une altérité à partir de laquelle elle découvre en même temps sa finitude et l'excès de ce qu'elle peut devenir. Dès lors, saint Augustin peut permettre au personnalisme contemporain de comprendre autrement l'essence relationnelle de la personne humaine que comme une inter-subjectivité, c'est-à-dire autrement que comme un rapport entre des subjectivités se constituant d'abord par elles-mêmes. Ainsi, l'essence relationnelle de la personne peut, avec l'aide de saint Augustin, ne plus être séparée de son essence événementielle. L'origine prosopographique et non pas anthropologique de l'usage du terme de personne introduit l'idée que toute personne hu- 
maine est elle-même une réponse et se découvre dans la question “qui m’appelle?". Plus précisément, la personne humaine est une réponse parce qu'elle "répond à" plus que parce qu'elle "répond de". En effet, la personne humaine ne devient pour elle-même une question que parce qu'elle est mise en question par l'événement d'une altérité et non par le seul pouvoir de sa réflexion. Tout événement, en tant qu'il est au-delà de l'imaginable, du probable, reconduit la personne à l'inimaginable d'elle-même: elle devient à la fois ce qu'elle n'aurait pas pu imaginer devenir et ce qu'elle n'aurait pas pu devenir sans l'événement. De fait, le terme grec prosôpon a d'abord signifié la face ou le visage et même d'une façon encore plus générale l'“avant" d'un objet. Or, de ce point de vue, la personne est celle qui s'ouvre notamment à la venue en présence de l'autre personne: elle ne reçoit un visage que par la grâce d'un autre visage. Cela dit, l'événement de l'autre homme n'est pas que la cause occasionnelle de la réflexion sur soi, il ne fait pas que m’ouvrir à une potentialité de moi déjà là, mais il me fait véritablement devenir autre, il m’informe imprévisiblement.

Sans pouvoir exposer ici toute la phénoménologie contemporaine de l'événement engagée par Emmanuel Lévinas et Henri Maldiney, il s'agit avant tout d'indiquer que le retour à saint Augustin est ce qui peut permettre d'arracher à l'enlisement en soi d'un sujet qui voulant se produire lui-même finit par s'identifier à la représentation qu'il a de lui-même. Le devenir soi authentique est une libération de la transcendance et celle-ci n'est possible que par l'expérience d'une altérité radicale à soi qui m’appelle à être en m’appelant à ce que je ne suis pas. Ainsi l'ipséité comme réponse, l'ipséité responsive, qui n'est ni l'ipséité transcendantale ni l'ipséité existentiale, présuppose une transcendance dans la passivité qui fait que chaque personne dans sa singularité incommunicable est une certaine façon d'être à l'altérité, de la recevoir. Il s'agit donc de retrouver aujourd'hui cette dimension relationnelle et responsive de la personne qui ne doit justement pas être comprise simplement comme un étant qui a des relations et qui répond. Les analyses augustiniennes montrent que je suis une relation et une réponse avant toute ouverture à mes possibilités les plus propres et que l'accueil de l'événement ne doit pas être interprété d'une façon ontique comme la capacité d'un sujet à s'approprier une altérité, de la ramener à soi pour en faire un objet de son monde environnant.

L'ipséité à laquelle nous ouvre saint Augustin est une identité d'exil, puisque l'appel qui advient hors de toute attente possible met la personne en demeure de renaître, de se transformer, ou plus précisément de se laisser transformer par ce qui la touche de telle sorte qu'elle ne se possède elle-même qu'en se sachant appartenir à autre chose qu'elle. En conséquence, retourner à l'invention augustinienne de la personne peut permettre à la métaphysique contemporaine de s'affranchir véritablement du concept vulgaire de personne selon lequel elle est 
soit un simple agrégat de déterminations contingentes, un simple nœud de relations infinies, soit un sujet qui se soumet lui-même à la loi de la raison. Contre tout stoïcisme, saint Augustin enseigne que la seule vraie patience trouve son origine dans l'endurance d'une altérité qui me conduit à m'endurer moi-même dans cette rencontre. Parce que l'Idipsum est le Verbe, l'ipséité humaine est une réponse et ce qui nous prend est aussi ce qui nous donne d'être en prise sur le monde au lieu de fuir le monde et de se fuir dans la citadelle d'une intériorité dans laquelle nous nous emmurons. Comme le dit Henri Maldiney, être une personne c'est exister «au péril du rien» ${ }^{29}$, c'est-à-dire par cette fidélité à ce qu'on ne peut pas objectiver. Si la personne est un voir, ce voir est plus réceptif que productif, il n'est pas l'acte d'un sujet, d'un esprit ou d'une conscience, mais bien l'acte d'une personne qui par tout son être, c'est-à-dire aussi par son corps, rend visible en recevant la lumière de l'être lui-même. Les Confessions et la Trinité donnent à comprendre que l'on n'est une personne qu'à être ouvert à l'aurore du visage de Dieu. Ces analyses augustiniennes libèrent du même coup de la représentation moderne de la personne comme un Hercule se produisant luimême et produisant le monde. Contre cette idée d'une création de soi par soi, saint Augustin nous fait redécouvrir l'idée d'une identité d'exode faite d'une ouverture à la révélation originaire du monde, du prochain et de Dieu. La personne est alors l'être qui sait écouter la parole discrète des choses et des autres et qui reçoit dans cette écoute sa stature, sa tenue. Telle est la possibilité à la fois commune et individuante de se posséder dans l'écoute, de se tenir soi-même en se laissant requérir à la vigilance par ce qui s'annonce à nous. Il n'est alors possible d'être soi que dans la conversion de son intériorité finie en un lieu de révélation du monde, des autres et de Dieu, sans prétendre à une appropriation.

Il est urgent au plus haut point de redonner au terme de personne la densité d'un concept et c'est pourquoi saint Augustin peut nous apprendre à redécouvrir cette essence relationnelle et responsive de la personne pour cesser de la comprendre comme une chose douée de réflexion. La personne ne redécouvre sa vocation responsive que dans l'abandon de toute identité construite, de toute crispation sur une image d'elle-même, pour retrouver un avenir dans l'ignorance de sa place et dans l'écoute de ce qui la requiert. Il n'est possible d'être soi qu'à renoncer à déterminer par soi seul sa place dans le monde pour accepter de vivre dans l'injonction des choses mêmes, dans cette écoute qui porte en elle une obéissance. A partir de saint Augustin il est possible de retrouver cette idée qui est à l'origine du concept chrétien de personne: mon être n'est pas là où je suis, mais là où se trouve l'injonction des choses. Dieu bien sûr, le visage de mon

29 H. Maldiney, Penser l'homme et la folie, Millon, Grenoble 1991, p. 357. 
prochain, mais la plus humble des choses également peuvent énoncer le "tu dois" et me faire advenir à moi-même en me mettant en exil. L'homme ne devient ainsi une personne qu'à répondre de tout son être à ce qui le met en exil et c'est pourquoi saint Augustin peut donner à la métaphysique contemporaine de repenser la personne comme témoin ${ }^{30}$, de comprendre à nouveau le témoignage comme le sens d'être de la personne.

30 Voir les perspectives ouvertes par le n. 88 de la revue «Philosophie» intitulée Le témoignage, Minuit, hiver 2005. 